\title{
SECADO NATURAL Y SOLAR DE HONGOS COMESTIBLES SILVESTRES DE LA REGION SAN MARTÍN
}

\author{
Oscar Mendieta Taboada * \\ Mari Medina Vivanco*
}

\section{RESUMEN}

En el presente trabajo se ha efectuado el secado de tres especies de hongos comestibles silvestres Auricularia fuscosuccinea, Auricularia delicata y Pleurotus ostreatus, mediante dos formas: secado natural (radiación solar directa) y empleando secador solar indirecto. La velocidad de secado fue mayor para el secado mediante radiación solar directa. Las isotermas de adsorción trazadas para los hongos deshidratados indican valores de 0,20, 0,35 y 0,44 de Aw para A. fuscosuccinea, A. delicata y $P$. ostreatus, respectivamente.

Palabras claves: Secado solar, hongos comestibles, A. fuscosuccinea, A. delicata, P. ostreatus, isotermas.

\section{INTRODUCCION}

Los hongos siempre fueron considerados un alimento especial y se les ha atribuido toda clase de propiedades. Para los faraones, por ejemplo, eran un manjar selecto; los griegos creían en cambio que los hongos daban fuerza a los guerreros; los romanos pensaban que eran un alimento de los dioses y, por último, para los chinos los hongos eran un alimento que mejoraba y conservaba la salud (Ekwalanga, et al 1990).

De otro lado, la escasez de alimentos es un problema que viene azotando desde hace muchos años a la población mundial, siendo las dietas consumidas bastante pobres en cuanto a su contenido energético y proteico (Anónimo 1985). Asimismo, se sabe que los bosques son una fuente importante de alimentos vegetales de gran valor nutritivo y comercial, pero poco conocidos.

Facultad de Ingeniería Agroindustrial. Universal Nacional de San Martín. Casilla Postal 239-Tarapoto. 
Entre estos tenemos a los hongos comestibles silvestres, de diferentes especies, que en nuestra Región son conocidos como "callampas", los cuales poseen un valor nutritivo relativamente elevado comparado con alimentos vegetales de consumo cotidiano Collazos, C. (1986). A estos hongos, propios de nuestra Región, no se les ha brindado la importancia debida, a excepción de algunos trabajos efectuados con apoyo del Programa de Manejo Ambiental - PEHCBM Mendieta, O. y J . Abad. (1991).

Los hongos constituyen una alternativa a las caras proteínas animales. Doscientos veinticinco gramos de hongos botones cultivados suministran $6 \mathrm{~g}$. de proteínas y 62 calorías. El contenido en grasas de los hongos incluye toda clase de lípidos, pero ellos tienen una cantidad del ácido graso esencial (el linoleico) muy superior a la que existe en otros vegetales. Los hongos son también una buena fuente de vitaminas, especialmente tiamina, riboflavina, niacina y biotina. Además contienen importantes cantidades de fósforo, potasio y sodio, al igual que otras menores de calcio y hierro Ekw alanga, et al (1990).

Tomando en cuenta los resultados obtenidos por algunos investigadores se pueden citar los siguientes datos sobre la composición de los hongos frescos: agua, 73 - 91\%; sustancias nitrogenadas, 4 - 9\%; grasas, 0,2 - 0,5\%; extracto no nitrogenado, 2 - 10\%; celulosa, 0,5 $5 \%$ y cenizas, $0,5-2 \%$ Collazos, C. (1986).

El Perú invierte aproximadamente $350 \quad 000$ dólares al año importando hongos de Chile y China. Esto debido al hecho que el cultivo de hongos comestibles no ha sido popularizado todavía, lo cual hace que estos alcancen un alto precio en el mercado.

Algunas de las especies de hongos de los bosques sanmartinenses como A. fuscosuccinea, P. ostreatus, A. delicata y A. polytricha, son similares a las especies importadas, en estado seco, de China y Filipinas Mendieta, O. y J. A bad (1991). Puesto que en los bosques de la Región San Martín crecen hongos comestibles, en forma silvestre, y al no existir una tecnología adecuada de conservación de los cuerpos fructíferos recolectados, se efectuó el presente trabajo con los siguientes objetivos:

Contribuir a la conservación de las zonas boscosas de la Región San Martín mediante el aprovechamiento de productos alimenticios no tradicionales.

Trazar curvas de secado natural y solar de tres especies de hongos de la Región.

Determinar la humedad monomolecular de las tres especies de hongos deshidratados. 


\section{MATERIALES Y METODOS}

\section{LUGAR DE EJ ECUCION}

La recolección de los cuerpos fructíferos de hongos comestibles silvestres fue efectuada en zonas boscosas y húmedas de la Región San Martín, aledañas a la ciudad de Tarapoto. Las especies consideradas fueron: Auricularia fuscosuccinea, Auricularia delicata y Pleurotus ostreatus, cuyos cuerpos fructíferos se presentan en la Figura 1.

En los laboratorios de la Universidad Nacional de San MartínTarapoto, fueron realizadas las experiencias de secado y trazado de isotermas de adsorción, en el período comprendido entre los meses de abril y octubre de 1993.

\section{MATERIALES Y EQUIPOS}

\section{Materiales}

Campanas desecadoras

Placas de petri

Cloruro de litio

Acetato de potasio

(Riedel de Haen, p.a.)

Cloruro de magnesio

(Riedel de Haen, p.a.)

Dicromato de sodio

(Riedel de Haen, p.a.)

Nitrito de sodio

(Riedel de Haen, p.a.)

Cromato de potasio

(Riedel de Haen, p.a.)

Nitrato de potasio

(Riedel de Haen, p.a.)

Cajas térmicas de poliuretano

(Riedel de Haen, p.a.)

Bolsas plásticas y de papel

Cuchillos

Material de vidrio (diversos)

\section{Equipos}

Estufa Memmert U-30

Balanza analítica Sartorius

Balanza eléctrica Owalabor

Selladora de bolsas Arno

Psicrómetros 0 a $60^{\circ} \mathrm{C}$

Termómetros de mercurio 0 a $100^{\circ} \mathrm{C}$

Secador solar de cabina, capacidad $25 \mathrm{~kg}$. 
FIG. I HONGOS COMESTIBLES SILVESTRES CONSIDERADOS EN EL ESTUDIO

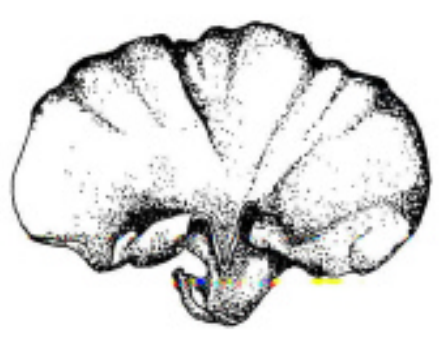

a) Auricularia fuscosuccinea (Core superior)

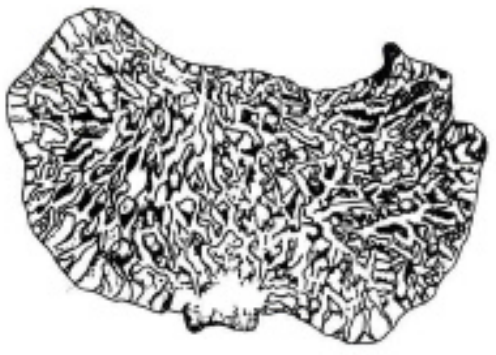

b) $\frac{\text { Auricularia }}{\text { (Cara inferior) }}$

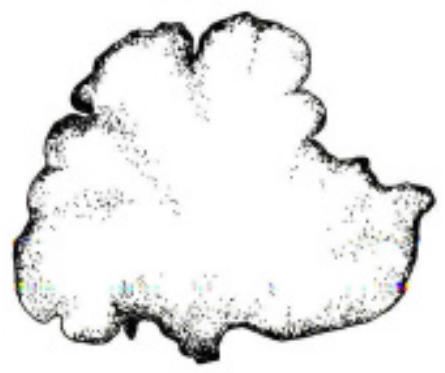

c) Pleurotus ostreatus (Cara superior)

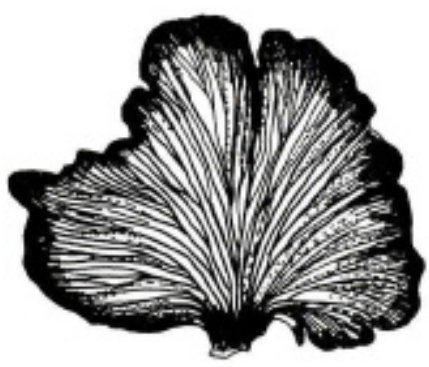

d) Pleurotus ostreatus (Cara inferior) 


\section{METODOS}

\section{Secado}

Se ensayaron dos formas de secado: secado natural (radiación solar directa) y secado solar indirecto (empleando secador solar), trazándose curvas de secado (peso vs. tiempo) para ambas formas de secado y para cada especie de hongo considerada. La muestra a deshidratar estuvo constituida por $1 \mathrm{~kg}$. de hongos frescos, por cada especie y ensayo, efectuándose tres repeticiones. Los cuerpos fructíferos fueron lavados con agua potable, para eliminar materias extrañas y oreados a continuación. Durante el proceso de secado fueron controladas las temperaturas de bulbo seco y bulbo húmedo, además del tiempo de secado y pérdida de peso del producto (Carbonel, et al 1985, Espeleta et al 1983 y Galarza 1986).

La determinación de humedad inicial y final de los hongos fue realizada mediante la técnica descrita por la A.O.A.C. 1970. El rendimiento del proceso, expresado en porcentaje, fue determinado relacionando el peso inicial con el peso final y multiplicando el resultado por 100.

\section{Isotermas de adsorción}

Con la finalidad de conocer las características higroscópicas de los hongos deshidratados, se trazó la isoterma de adsorción de los mismos, empleando el método estático gravimétrico descrito por Stitt (1958), citado por Martínez, 1967, empleando soluciones salinas saturadas. Las solu-ciones salinas utilizadas y la humedad relativa decimal de las mismas se presenta en el Cuadro 1.

\section{Humedad monomolecular}

La humedad monomolecular fue determinada mediante la ecuación de BET (Martínez, 1967), la cual fue ajustada a los datos experimentales empleando el paquete estadístico Statgraf 4.0 


\section{Cuadro 1}

Soluciones salinas saturadas utilizadas para fijar isotermas de adsorción

\begin{tabular}{|l|c|}
\hline \multicolumn{1}{|c|}{ SOLUCIONES SATURADAS } & HUMEDAD RELATIVA* \\
\hline Cloruro de litio & 0,110 \\
Acetato de potasio & 0,220 \\
Cloruro de magnesio & 0,325 \\
Dicromato de sodio & 0,503 \\
Nitrito de sodio & 0,640 \\
Cromato de potasio & 0,855 \\
Nitrato de potasio & 0,930 \\
\hline
\end{tabular}

* Valores a temperatura ambiente (25 - 35?C)

\section{RESULTADOS Y DISCUSION}

El contenido de humedad de la materia prima utilizada (hongos frescos), se presenta en el Cuadro 2. Puede notarse que el contenido de humedad de las tres especies mencionadas es bastante próximo a $90 \%$, corroborando lo reportado en la literatura (Collazos, C. 1986).

\section{Cuadro 2}

Contenido de humedad de hongos frescos

\begin{tabular}{|l|c|}
\hline \multicolumn{1}{|c|}{ ESPECIE } & HUMEDAD PORCENTUAL* \\
\hline Auricularia fuscosuccinea & 89,01 \\
Auricularia delicata & 90,20 \\
Pleurotus ostreatus & 89,70 \\
\hline
\end{tabular}

* En base húmeda 


\section{SECADO}

La evolución de temperaturas de bulbo seco y bulbo húmedo, durante el proceso de secado, se presentan en la Figura 2. Pueden apreciarse cambios en las mismas debido a la variación horaria y a la presencia de nubosidad. Ambas temperaturas alcanzan su valor más alto (48 - 37?C, para T.b.s. y T.b.h. respectivamente) en el interior de la cámara de secado a las 14:00 horas (hora solar); lo cual se debió a que en este momento la radiación solar incidente en el colector del secado solar alcanzó su valor más elevado.

Los resultados del proceso de secado de $A$. fuscosuccinea, $A$. delicata y $P$. ostreatus se muestran en las Figuras 3,4 y 5 respectivamente. En todas ellas pueden notarse que la pérdida de peso es más acentuada cuando el secado se realiza mediante radiación solar directa. Esto se debe probablemente a que el material a secar, al absorber la radiación solar incidente, aumenta su temperatura en una proporción mayor que las muestras colocadas en el secador solar indirecto, por lo cual la evaporación del agua en las mismas es más acentuada.

En los hongos secados con radiación solar directa, además de la mayor velocidad de secado, se presentó un mayor oscurecimiento debido posiblemente a pardeamiento no enzimático favorecido por la acción de los rayos ultravioleta presentes en la radiación solar; aparte que por no estar protegidos quedaban expuestos a la acción contaminante de insectos y al polvo.

El rendimiento luego del proceso de cada una de las especies de hongos, considerando un contenido de humedad final del $10 \%$, se presenta en el Cuadro 3. Puede apreciarse que el rendimiento es bajo, pero característico para productos deshidratados (Espeleta, et al 1983, Galarza, 1988 y Torres 1986). 
FIG. 2 VARIACION DE TEMPERATURAS EN EL SECADOR SOLAR.

- Tomperatura bulbo soco

^ Tomporatura bulbo húmedo

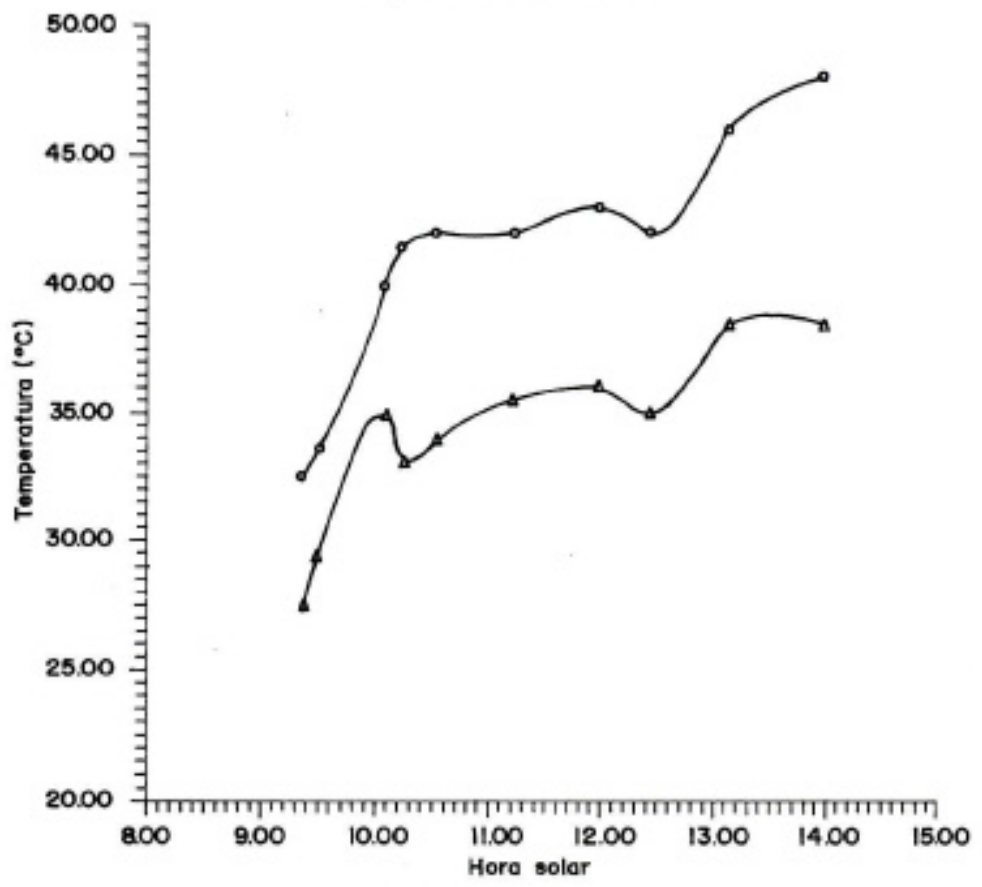


FIG. 3 CURVAS DE SECADO DE Auriculario fuscosuccinec

- Secodor solar

$\Delta$ Rodiación directa

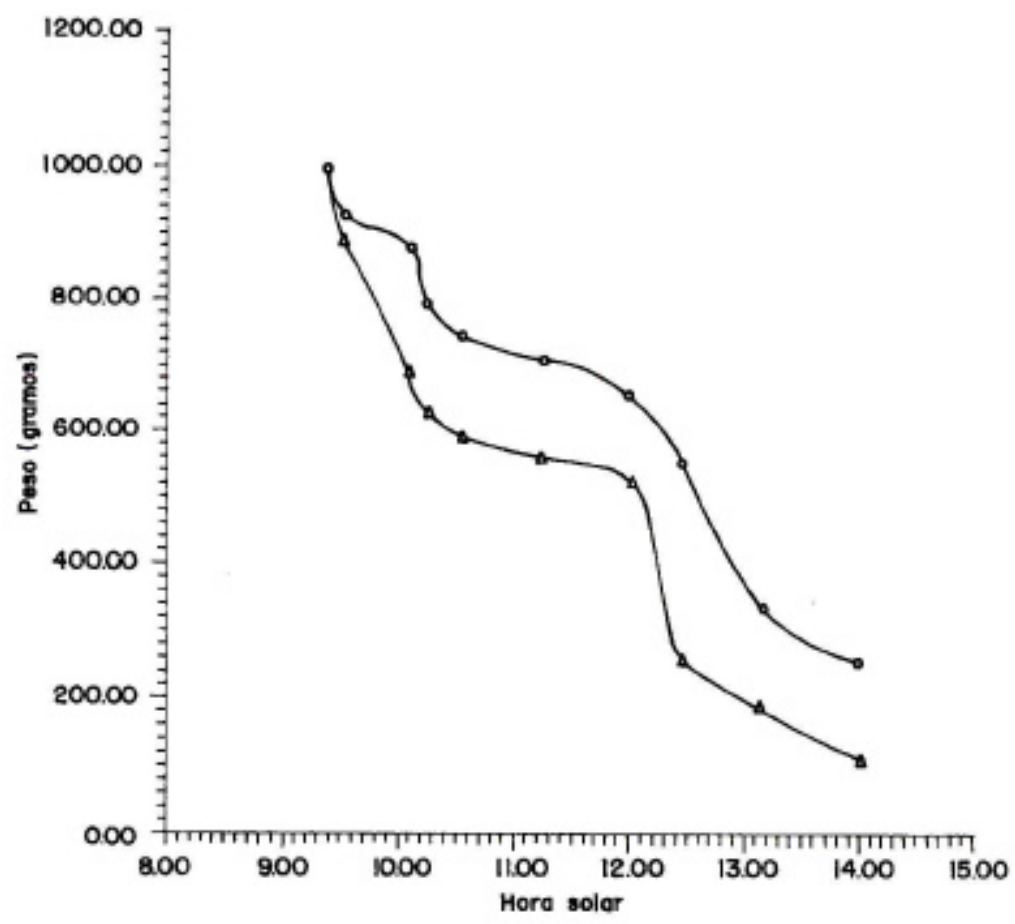


FIG. 4 CURVAS DE SECADO DE Auriculario delicato

- Secador solar

A Radiación directa

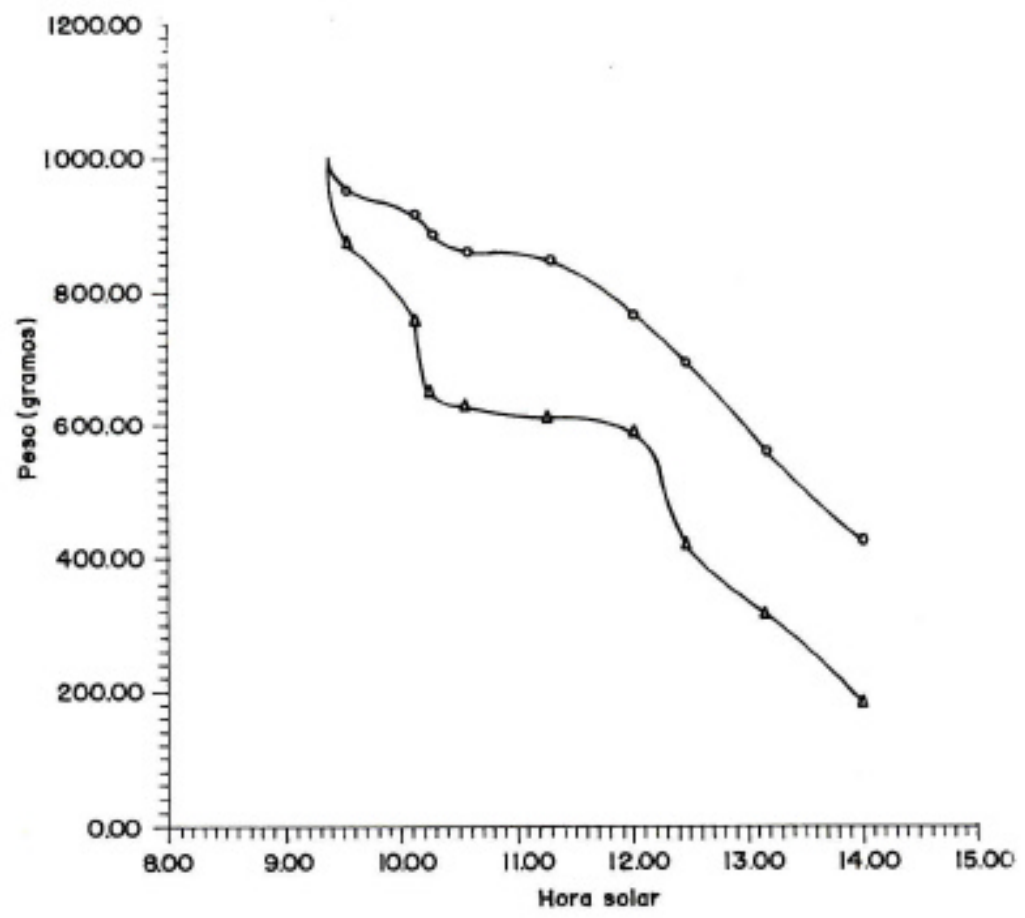


FIG. 5 CURVAS DE SECADO DE Pleurotus ostreatus

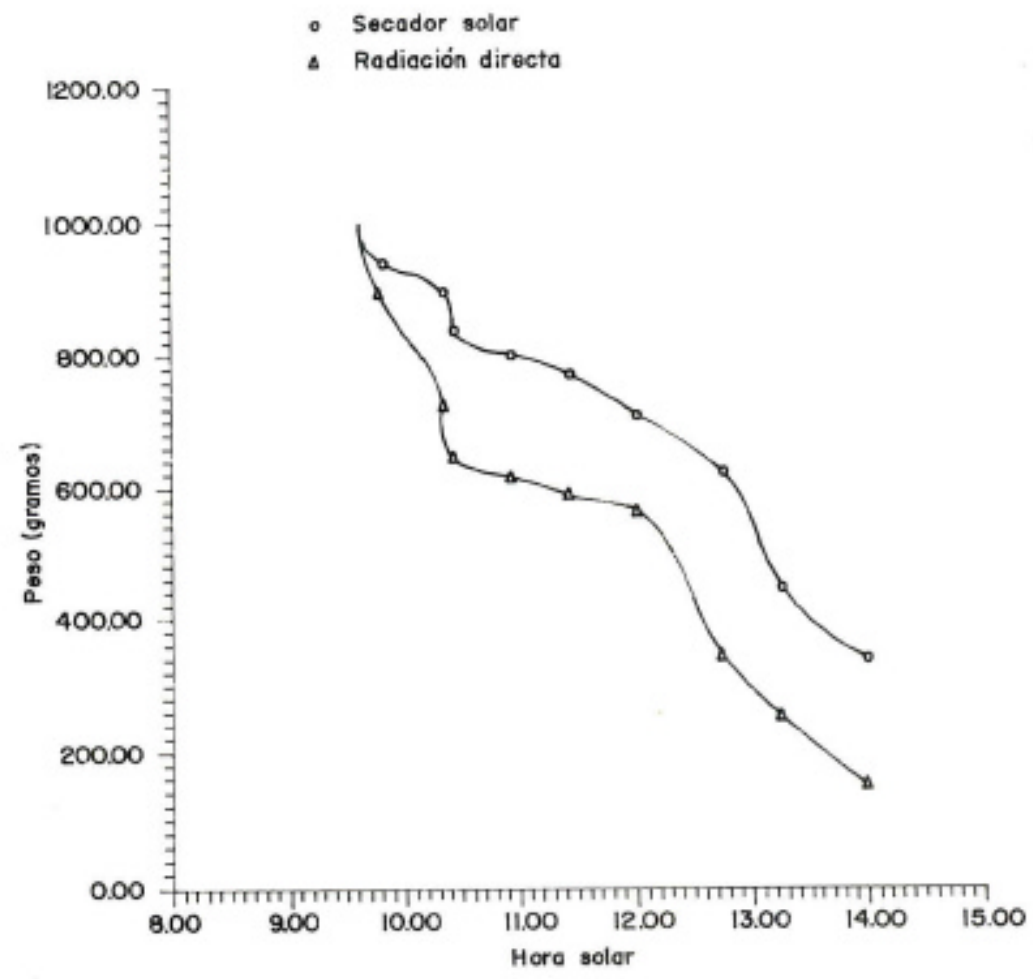




\section{Cuadro 3}

\section{Rendimiento de hongos deshidratados*}

\begin{tabular}{|l|c|}
\hline \multicolumn{1}{|c|}{ ESPECIE } & RENDIM IENTO (\%) \\
\hline Auricularia fuscosuccinea & 12,21 \\
Auricularia delicata & 10,88 \\
Pleurotus ostreatus & 11,44 \\
\hline
\end{tabular}

* Humedad final: $10 \%$ en base húmeda

\section{ISOTERMA DE ADSORCION}

La isoterma de adsorción para cada una de las especies de hongos deshidratadas se presenta en la Figura 6. A partir de estas curvas puede determinarse que el valor de actividad de agua (Aw), para el contenido de humedad luego del secado (10\%) fue de 0,20, 0,35 y 0,44 para A. fuscosuccinea, $A$. delicata y $P$. ostreatus, respectivamente; mientras que la humedad monomolecular determinada mediante la ecuación de BET, para Actividades de agua comprendidas entre 0,11 y 0,50 fue 7,93, 7,41 y 5,62 g. de agua/100 g. de materia seca. En base a los valores de Aw mencionados anteriormente se concluye que los hongos deshidratados, por ser materiales muy higroscópicos, deben ser protegidos con un empaque adecuado que impida el paso de vapor de agua y, en consecuencia, el deterioro. 
FIG. 6 ISOTERMA DE ADSORCION DE HONGOS DE LA REGION SAN MARTIN (Temperatura ambiente : $25-35^{\circ} \mathrm{C}$ )

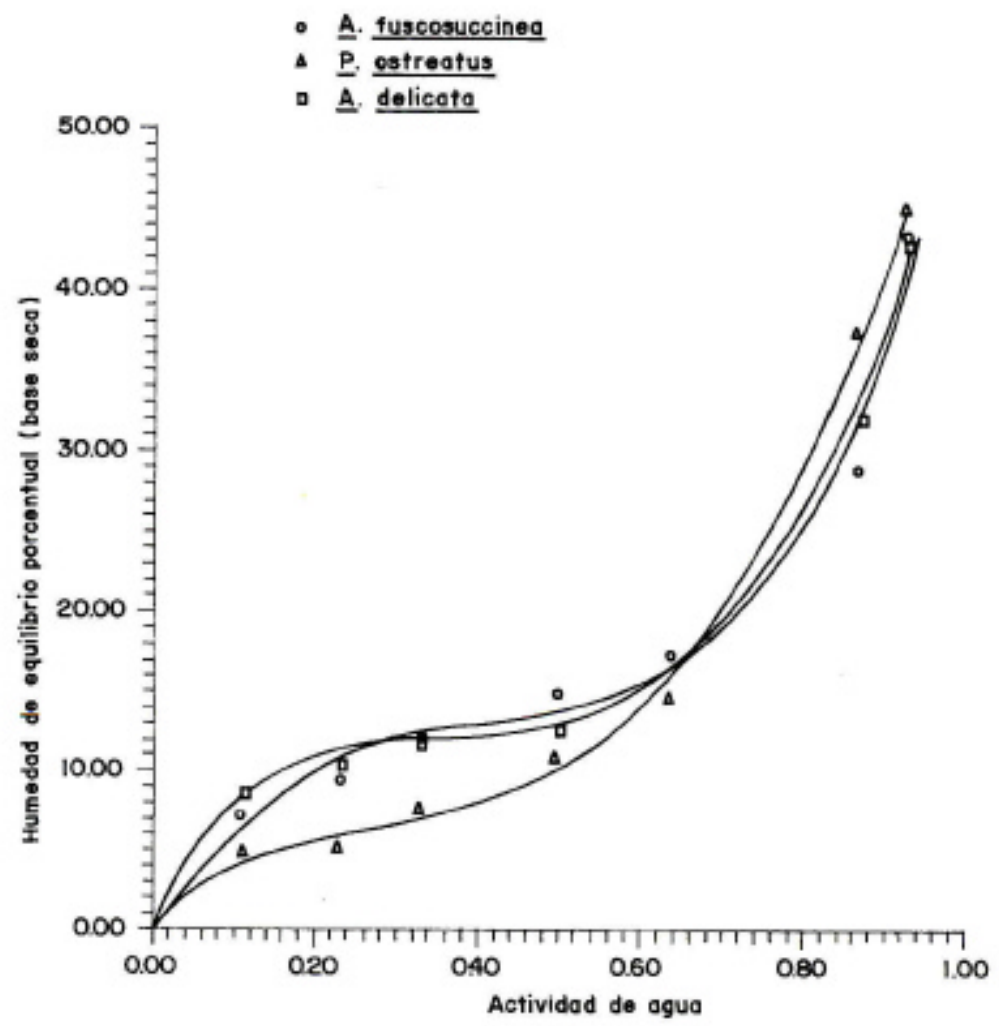




\section{CONCLUSIONES}

1. La deshidratación constituye una tecnología adecuada para la conservación de hongos comestibles silvestres, debiendo ser complementado con el empacado respectivo, pues se trata de materiales higroscópicos.

2. El secado por radiación solar directa fue más rápido que el secado realizado empleando secador solar indirecto, para las tres especies de hongos utilizados.

3. La humedad monomolecular fue de $7,93,7,41$ y $5,62 \mathrm{~g}$. de agua/100 g. de materia seca, para A. fuscosuccinea, A. delicata y P. ostreatus, respectivamente.

\section{AGRADECIMIENTO}

Al Consejo Nacional de Ciencia y Tecnología por el apoyo económico brindado para la realización del presente trabajo. Al Ingo M. Fernando Coronado J., por su valiosa colaboración durante el desarrollo de esta investigación.

\section{BIBLIOGRAFIA}

ANONIMO, 1985. "La Silvicultura y la seguridad alimentaria". Departamento de Montes, en FAO, Vol. 37, p. 4-13.

A.O.A.C. 1970. "Official Methods of Analysis", en Association of Official Agriculture Chemists. 11a. Ed.

CARBONEL, J .; F. PIÑAGA, J . PEÑA.1985."Deshidratación de alimentos por energía solar. IV. Ensayos con zanahorias", en Rev. Agroquim. Tecnol. Aliment. 25 (1): 87-94 
COLLAZOS, C. 1986. "Tabla de composición de los alimentos peruanos", Instituto de Nutrición, Lima, Perú, 50 p.

EKWALANGA, M. y M.C. COMTE 1990. "Una industria que puede crecer como los hongos", en Rev. CERES, Vol. 126 (Nov.-Dic.): 4749.

ESPELETA, I, M. KOSS y R. GARCIA. 1983. "Secado solar de hongos comestibles", en: Ing. y Ciencia Química 7 (3-4): 35-41.

GALARZA, C. 1986. "Secado solar de cebolla en Tacna". en II Seminario de Secado Solar, Cusco.

MARTINEZ, F. 1967. "Estudio de la relación humedad actividad de agua en algunos alimentos", en Anales científicos UNA (3-4): 191-205.

MENDIETA, O. Y J. ABAD, 1991. "dentificación y Análisis bromatológico de dos especies de hongos comestibles silvestres", en Programa de Manejo Ambiente - PEHCBM, 10p.

TORRES, R. 1986."Secado solar de frutas en Cajamarca", en ॥ Seminario de Secado Solar, Cusco. 